\title{
Murrangatin suppresses angiogenesis induced by tumor cell-derived media and inhibits AKT activation in zebrafish and endothelial cells
}

This article was published in the following Dove Press journal:

Drug Design, Development and Therapy

\section{Weiqing Longl,* \\ Mingjun Wang ${ }^{2, *}$ \\ Xiongming Luo $^{3}$ \\ Guixian Huang ${ }^{4}$ \\ Jianwen Chen ${ }^{5}$}

'Department of Clinical Laboratory,

The First Affiliated Hospital, Sun

Yat-sen University, Guangzhou

510080, China; ${ }^{2}$ Department of Pharmacy, The First Affiliated Hospital, Sun Yat-sen University, Guangzhou 510080 , China; ${ }^{3}$ CAS Key Laboratory of Tropical Marine Bio-resources and Ecology, South China Sea Institute of Oceanology, Chinese Academy of Sciences, Guangzhou 5I030I, China; ${ }^{4}$ Department of Emergency, The First Affiliated Hospital, Sun Yat-sen University, Guangzhou 510080, China; ${ }^{5}$ School of Pharmaceutical Sciences, Sun Yat-sen University, Guangzhou 5I0006, China

*These authors contributed equally to this work
Correspondence: Xiongming Luo CAS Key Laboratory of Tropical Marine Bio-resources and Ecology, South China Sea Institute of Oceanology, Chinese Academy of Sciences, Guangzhou 51030I, China

Email xiongmingluo@scsio.ac.cn

Jianwen Chen

School of Pharmaceutical Sciences,

Sun Yat-sen University,

Guangzhou 510006, China

Email573016880@qq.com

\begin{abstract}
Introduction: Lung cancer is a major cancer type and a leading cause of cancer-related death. Angiogenesis plays a crucial role in lung cancer pathogenesis and its inhibition is beneficial to patients.
\end{abstract}

Materials and methods: Murrangatin, a natural product, can inhibit the proliferation of lung cancer cells, so herein we investigated its anti-angiogenic effects in transgenic zebrafish TG (fli1: EGFP) and in lung cancer cell-induced angiogenesis in human umbilical vein endothelial cells.

Results: We found that murrangatin strongly inhibited the growth of subintestinal vessels in zebrafish embryos and tumor conditioned media-induced angiogenic phenotypes including cell proliferation, cell invasion, cell migration, and tube formation. Additionally, murrangatin greatly attenuated conditioned medium-induced AKT phosphorylation, but not extracellular signal-regulated kinase $1 / 2$ phosphorylation.

Discussion and conclusion: These findings indicate that murrangatin can inhibit tumorinduced angiogensis, at least in part through the regulation of AKT signaling pathways. Murrangatin may, therefore, be a potential candidate for the development of new anti-lungcancer drugs.

Keywords: anti-angiogenesis, murrangatin, conditioned medium, zebrafish, HUVECs, AKT

\section{Background}

Lung cancer is a major type of cancer and a leading cause of cancer-related death. Non-small-cell lung cancer (NSCLC) accounts for more than $80 \%$ of all lung cancer cases. ${ }^{1}$ Although the surgical removal of tumors is the best option for patients with early stage NSCLC, $70 \%-80 \%$ of patients, particularly those with advanced cancer, cannot undergo surgery because of poor pulmonary function or metastatic disease. Thus, chemotherapy is the first-line treatment option for patients with NSCLC. Unfortunately, standard chemotherapy for NSCLC based on cytotoxic agents often causes serious side effects and chemoresistance. Therefore, there is an urgent need to develop new drugs with minimal side effects for NSCLC treatment.

Angiogenesis is a complex process that plays an important role in the growth and metastasis of lung cancer. It is regulated by a number of factors, including the phosphoinositol 3-kinase (PI3K)/AKT and extracellular signal-regulated kinase (ERK) signaling pathways. Both AKT and ERK contribute to tumor-associated angiogenesis, whereas the downregulation of AKT or ERK pathways inhibits tumor growth. ${ }^{3,4}$ Cancer cells also secrete pro-angiogenic factors that enhance angiogenesis in NSCLC, while microvessel development has been directly linked to tumor maturation and tumor 
metastasis in NSCLC. ${ }^{2}$ Given the importance of signaling pathways in regulating tumor growth and angiogenesis, the development of therapeutic drugs targeting PI3K/AKT or ERK signaling pathways and the inhibition of angiogenesis are attractive strategies for cancer treatment.

Murrangatin is a natural product derived from a marine plant that was shown to inhibit the growth of the lung cancer cell line A549, indicating its anti-cancer properties. ${ }^{5}$ In this study, we investigated the potential inhibitory action of murrangatin on angiogenesis in zebrafish and in human umbilical vein endothelial cells (HUVECs). We found that murrangatin inhibited cancer-associated angiogenesis in HUVECs. Meanwhile, AKT phosphorylation was significantly decreased in murrangatin-treated HUVECs, suggesting that the antiangiogenetic property of murrangatin is mediated through modulation of the AKT signaling pathway. Given that angiogenesis plays an important role in NSCLC, the present study provides a rationale for future development of this compound as a potential drug in NSCLC treatment.

\section{Materials and methods}

\section{Preparation of murrangatin}

We prepared the murrangatin using commercially purchased reagents and solvents. High-performance liquid chromatography (HPLC) was used to repurify methanol. The Bruker DRX-500 spectrometer (Bruker, Bremen, Germany) was used to detect nuclear magnetic resonance spectra with SiMe4 (Cambridge Isotope Laboratories, Tewksbury, MA, USA) as the internal standard. The VG Auto Spec-3000 MS spectrometer (Bruker) was used to perform high-resolution electrospray ionization mass spectrometry. Thin layer chromatography was conducted on precoated silica gel $\mathrm{G}$ plates (Qingdao Haiyang Chemical Plant, Qingdao, China) to separate murrangatin. Plates were sprayed with $50 \%$ $\mathrm{H}_{2} \mathrm{SO}_{4}$ solution and heated to allow the visualization of spots. Semi-preparative reversed-phase HPLC was conducted on ODS columns (YMC-Pack ODS-5-A, $250 \times 10 \mathrm{~mm}, 5 \mu \mathrm{m}$; YMC, Kyoto, Japan) using the $\mathrm{CH}_{3} \mathrm{OH}-\mathrm{H}_{2} \mathrm{O}$ solvent system as eluents. The fraction and purity of murrangatin were examined using the Waters 600 HPLC system (Waters, Voorhees, NJ, USA) with a Waters 996 photodiode array detector (Waters).

\section{Isolation of murrangatin}

The isolation and purification of murrangatin was conducted at the CAS Key Laboratory of Tropical Marine Bio-resources and Ecology (South China Sea Institute of Oceanology, Chinese Academy of Sciences, Guangzhou, China).
The purity of the compound was $>98 \%$. Dimethyl sulfoxide (DMSO) was used to dissolve murrangatin, which was kept as a stock solution at $-20^{\circ} \mathrm{C}$. PBS was used to dilute the stock to achieve the desired concentration before use.

\section{Anti-angiogenesis in zebrafish embryos}

The transgenic zebrafish cell line TG (fli1: EGFP), expressing eGFP in endothelial cells, was obtained from ZFIN (Eugene, OR, USA). ${ }^{6,7}$ Adult fish (3-12 months of age) were pairwise mated to produce embryos. Healthy embryos at the 1-4 cell stage were collected and transferred into a 96-well microplate. Test compounds $(100 \mu \mathrm{L})$ at different concentrations were added into each well and incubated at $28^{\circ} \mathrm{C}$ for $72 \mathrm{~h}$. The assessment of embryonic subintestinal vessels (SIVs) was conducted $72 \mathrm{~h}$ postfertilization as described previously. ${ }^{7}$ A digital camera (Diagnostic Instruments Inc., Sterling Heights, MI, USA) connected to an OLYMPUS IX71 fluorescence inverted microscope (Olympus, Tokyo, Japan) was used to capture SIV images at $100 \times$ magnification. NIH ImageJ software (version 1.43) was used to measure the SIV length. The assay was performed three times and each group contained 20-30 embryos.

\section{Cell culture and preparation of tumor conditioned medium (CM)}

The A549 lung cancer cell line and HUVECs were obtained from ScienCell (Carlsbad, CA, USA) and cultured at $37^{\circ} \mathrm{C}$ in DMEM (Invitrogen, Carlsbad, CA, USA) supplemented with $10 \%$ fetal bovine serum (FBS). Cells were maintained in a humidified atmosphere of $5 \% \mathrm{CO}_{2}$. To obtain CM, A549 cells were seeded at a density of $1 \times 10^{6}$ cells in a $100 \mathrm{~mm}$ dish containing DMEM with 10\% FBS overnight. The medium was then replaced with serum-free DMEM and incubated for $72 \mathrm{~h}$. CM was collected and filtered with a $0.2 \mathrm{~mm}$ filter and then stored at $-80^{\circ} \mathrm{C}$.

\section{Cell viability assay}

The MTT Cell Growth Kit (Chemicon, Temecula, CA, USA) was used to evaluate cellular viability according to the manufacturer's instructions. Briefly, HUVECs were seeded at a density of $1 \times 10^{5}$ cells $/ \mathrm{mL}$ in $100 \mu \mathrm{L}$ of medium per well of a 96-well microplate. Different concentrations of murrangatin and/or CM were added for $24 \mathrm{~h}$. Then, $10 \mu \mathrm{L}$ of $0.5 \mathrm{mg} / \mathrm{mL}$ MTT labeling reagent was added to each well for an additional $4 \mathrm{~h}$ incubation at $37^{\circ} \mathrm{C}$ with $5 \% \mathrm{CO}_{2}$ and $95 \%$ air (v/v) at $90 \%$ humidity. The purple formazan crystals produced were dissolved with $100 \mu \mathrm{L}$ of solubilization reagent per 
well for further quantitative measurement at an absorbance of $570 \mathrm{~nm}$ using a microplate spectrophotometer.

\section{Cell invasion, migration, and tube formation}

A transwell assay was conducted to investigate HUVEC invasion as previously described. ${ }^{8} \mathrm{~A} 10 \mathrm{~mm}$ tissue culture insert (transwell) with polycarbonate membrane ( $8 \mathrm{~mm}$ pores) and 24-well companion plate were used to evaluate the effect of murrangatin on HUVEC invasion. The upper and lower sides of the membrane were precoated with $1: 30(\mathrm{v} / \mathrm{v})$ and $1: 100(\mathrm{v} / \mathrm{v})$ of Matrigel ${ }^{\mathrm{TM}}$. Low serum $(1 \% \mathrm{FBS})$ medium was used to resuspend HUVECs, and a density of $5 \times 10^{4}$ cells was added to each insert in triplicate. Inserts were then deposited into the 24-well companion plate containing $500 \mu \mathrm{L}$ of $1 \%$ FBS medium with CM and the indicated concentration of murrangatin. After $8 \mathrm{~h}$ of incubation, inserts were removed and washed with PBS. Cotton swabs were used to remove noninvasive cells on the upper surface of the membrane. Inserts were then fixed in paraformaldehyde, stained with 4',6-diamidino-2-phenylindole, and mounted on microscope slides. A fluorescent inverted microscope with a CCD camera was used to take photographs of the invasive cells at $100 \times$ magnification. Metamorph Imaging Series software (Molecular Devices, Tokyo, Japan) was used to quantify the number of cells per insert. The invading cell number was normalized to the total cell number for each group.

The wound healing method was used to examine the migration of HUVECs as previously described. ${ }^{8}$ HUVECs were incubated at a density of $3 \times 10^{5}$ cells per well of a 24-well plate with complete medium at $37^{\circ} \mathrm{C}$ with $5 \% \mathrm{CO}_{2}$. After $24 \mathrm{~h}$, the medium was replaced with low serum $(0.5 \%$ FBS) medium to starve HUVECs for $24 \mathrm{~h}$. Next, a P200 pipette tip was used to create a "wound" in each well by scraping in a straight line. Photographs of three randomly selected areas along the scraped line were taken using a CCD camera attached to a fluorescent inverted microscope at 50× magnification. The medium was then replaced with fresh low serum (1\% FBS) medium containing $\mathrm{CM}$ and the indicated concentration of murrangatin. After $12 \mathrm{~h}$ of incubation, more photographs were taken of the selected areas and analyzed by Metamorph Imaging Series software. The average scraped area of each well was measured before and after treatment and used to calculate the percentage of wound closure area.

Growth factor-reduced Matrigel was used to detect endothelial tube formation as described previously. ${ }^{7}$ Briefly, growth factor-reduced Matrigel $(250 \mu \mathrm{L})$ was pipetted into 24-well culture plates that were maintained at $37^{\circ} \mathrm{C}$ for $30 \mathrm{~min}$ to allow Matrigel polymerization. HUVECs were then incubated at a density of $5 \times 10^{4}$ cells on the Matrigelcoated plates in low serum ( $1 \% \mathrm{FBS})$ medium with $\mathrm{CM}$ and the indicated concentration of murrangatin at $37^{\circ} \mathrm{C}$ for $8 \mathrm{~h}$. An inverted microscope was used to investigate the network-like structures at $50 \times$ magnification. Tube-like structures were defined as endothelial cord formations that were connected at both ends. NIH ImageJ software was used to quantitatively measure tube lengths. ${ }^{7}$

\section{Western immunoblot analysis}

HUVECs were seeded and incubated at a density of $2 \times 10^{5}$ cells in $60 \mathrm{~mm}$ dishes for 2 days. Culture medium was then replaced with fresh, reduced serum medium ( $2 \%$ FBS) and different concentrations of murrangatin for $24 \mathrm{~h}$. Cellular proteins were harvested and immunoblots were performed as previously reported. ${ }^{9}$ The antibodies used included antiAKT (catalog\# 9272; Cell Signaling Technology, Danvers, MA, USA), antiphospho-AKT (P-AKT, catalog\# 9271; Cell Signaling Technology), anti-total ERK1/2 (T-ERK1/2, catalog\# 4695S; Cell Signaling Technology), anti-phosphoERK1/2 (P-ERK1/2, catalog\# 9102; Cell Signaling Technology), and anti- $\beta$-actin (catalog\# 2565; Biosynthesis Biotechnology, Beijing, China).

\section{Statistical analysis}

Data are shown as mean \pm standard error of the mean (SEM). Analysis of variance followed by the Newman-Keuls multiple comparison test was used to detect differences among test groups (Prism 4.0, GraphPad Software Inc., San Diego, CA, USA). The $p$-values $<0.05$ were regarded as statistically significant.

\section{Results}

\section{Isolation and preparation of murrangatin}

Murrangatin as a monomeric coumarin was originally isolated from Murraya elongata ${ }^{10}$ and later from the plant Micromelum falcatum Tan (Lour.) in Hainan Island. Ten kilogram of air-dried material was extracted from Micromelum falcatum (Lour.) using 95\% EtOH; this was carried out three times. The aqueous residue was then extracted with $n$-hexane and EtOAc three times each, and the EtOAc extract (102 g) was separated using silica gel (1,300 g, 200-300 mesh) with solvents of increasing polarity: $10 \%-70 \%$ acetone in $n$-hexane. Fr.15 (1.35 g, eluted with $n$-hexane-acetone 6:4) was further fractionated using silica gel with chloroform-acetone (5:1). This obtained $118.0 \mathrm{mg}$ 


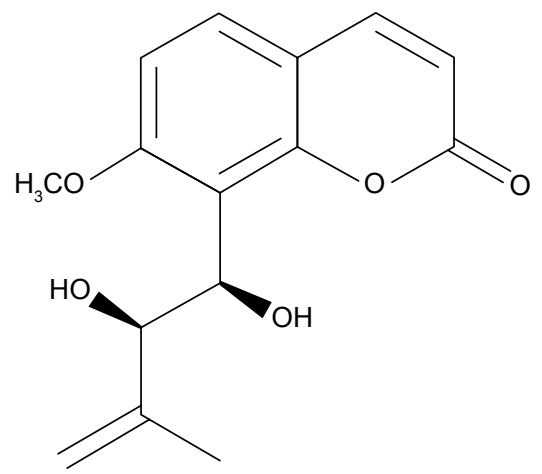

Figure I Chemical structure of murrangatin.

of murrangatin through semi-preparative HPLC purification $\left(250 \times 10 \mathrm{~mm}\right.$ i.d. $\left.5 \mu \mathrm{m}, \mathrm{MeOH} / \mathrm{H}_{2} \mathrm{O}, 55: 45\right)($ Figure 1$)$.

\section{Murrangatin inhibited the growth of SIVs in zebrafish embryos}

Murrangatin $(10,50$, or $100 \mu \mathrm{M})$ or vehicle (0.2\% DMSO) was incubated with zebrafish embryos for 72 h. Morphological changes are not different among different groups
(Figure 2). SIVs in vehicle-treated zebrafish embryos showed normal vessel development and formed a smooth basketlike structure (Figure 3A). In contrast, SIV formation was disrupted in murrangatin-treated zebrafish embryos. Murrangatin treatment dose-dependently reduced the length of SIVs in zebrafish embryos ( $p<0.01$, Figure 3B), while $100 \mu \mathrm{M}$ murrangatin completely blocked SIV formation (Figure 3A).

\section{Murrangatin inhibited CM-induced cell proliferation of HUVECs}

Endothelial cell proliferation is essential in tumor cellinduced angiogenesis. We, therefore, investigated whether murrangatin could inhibit tumor cell-induced endothelial cell proliferation. Given that tumor cells secrete pro-angiogenetic factors, media from A549 cell culture were used to induce proliferation of HUVECs. As shown in Figure 4, cell proliferation was significantly increased in HUVECs treated with CM compared with HUVECs suspended in serum-free DMEM. CM-induced cell proliferation was significantly

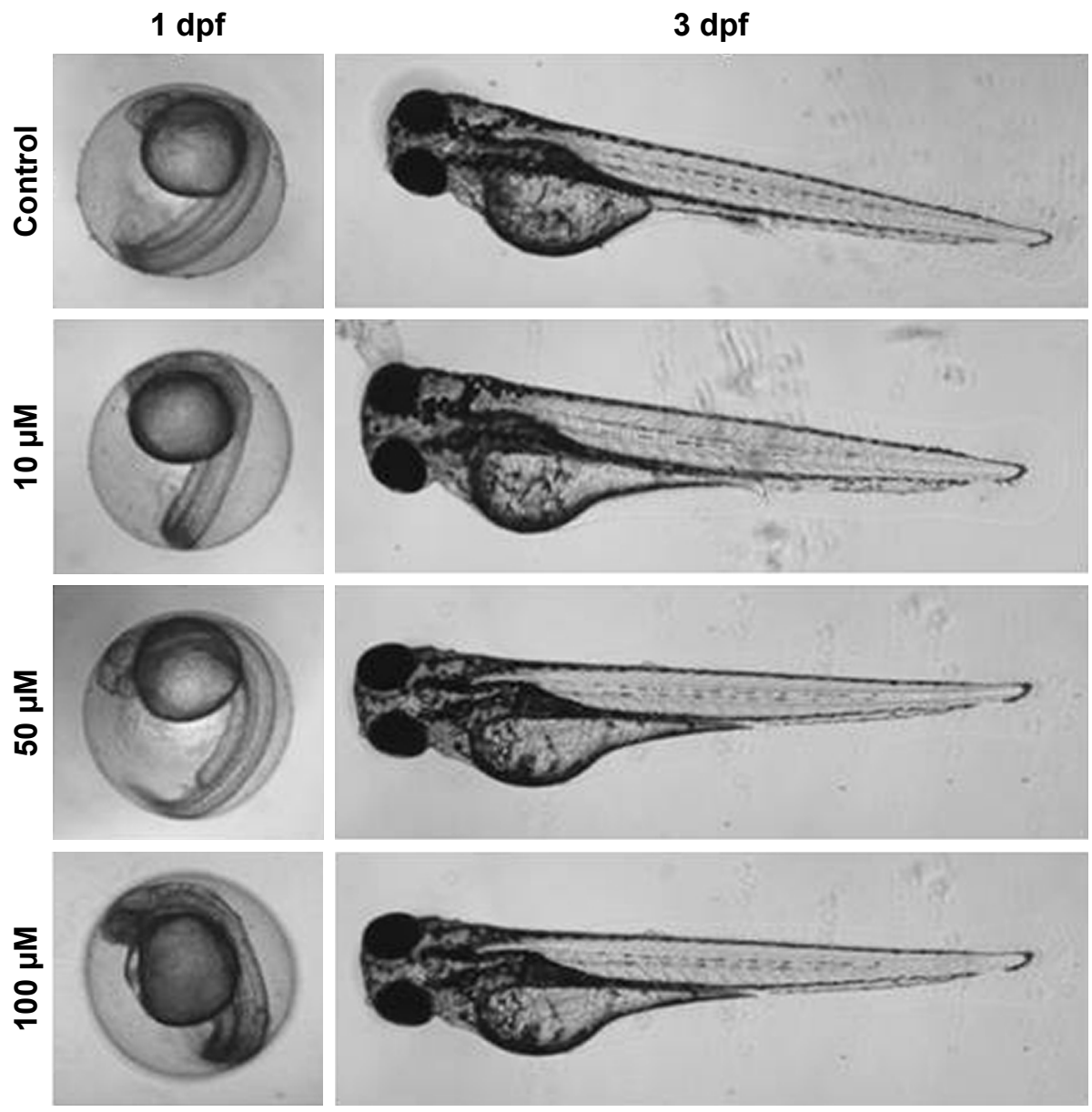

Figure 2 Effects of murrangatin on morphological changes of zebrafish embryo. Morphological changes are not different among different groups. Representative photographs of the morphological changes of the embryos treated with different concentrations of the murrangatin are shown. Experiments were performed in triplicate. 


\section{A}
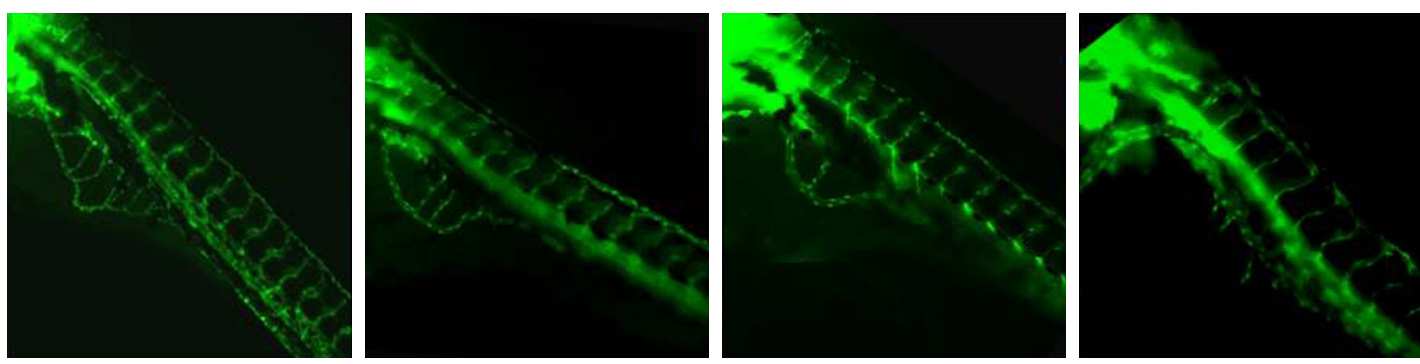

B

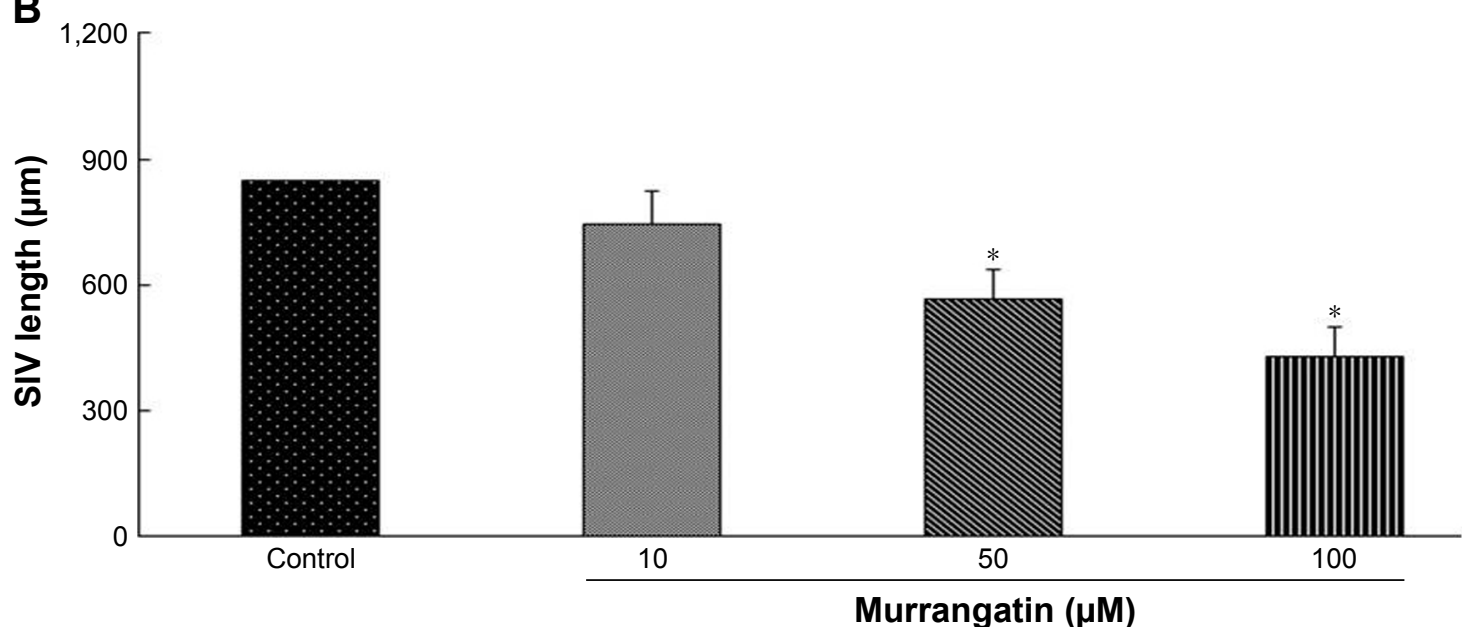

Figure 3 Murrangatin inhibited angiogenesis of SIVs in vivo. (A) Fluorescence images show the SIV morphology of 72-hpf TG (flil: EGFP) zebrafish embryos treated with DMSO or different concentrations of murrangatin. (B) Quantification of the SIV length in 72 hpf embryos in the vehicle control group and murrangatin-treated groups. Data are expressed as mean \pm SEM from three independent experiments ( ${ }^{p} p<0.01$ vs control; one-way ANOVA).

Abbreviations: SIV, subintestinal vessel; SEM, standard error of the mean; ANOVA, analysis of variance.

A

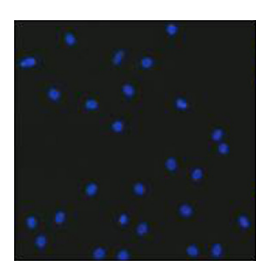

B

B
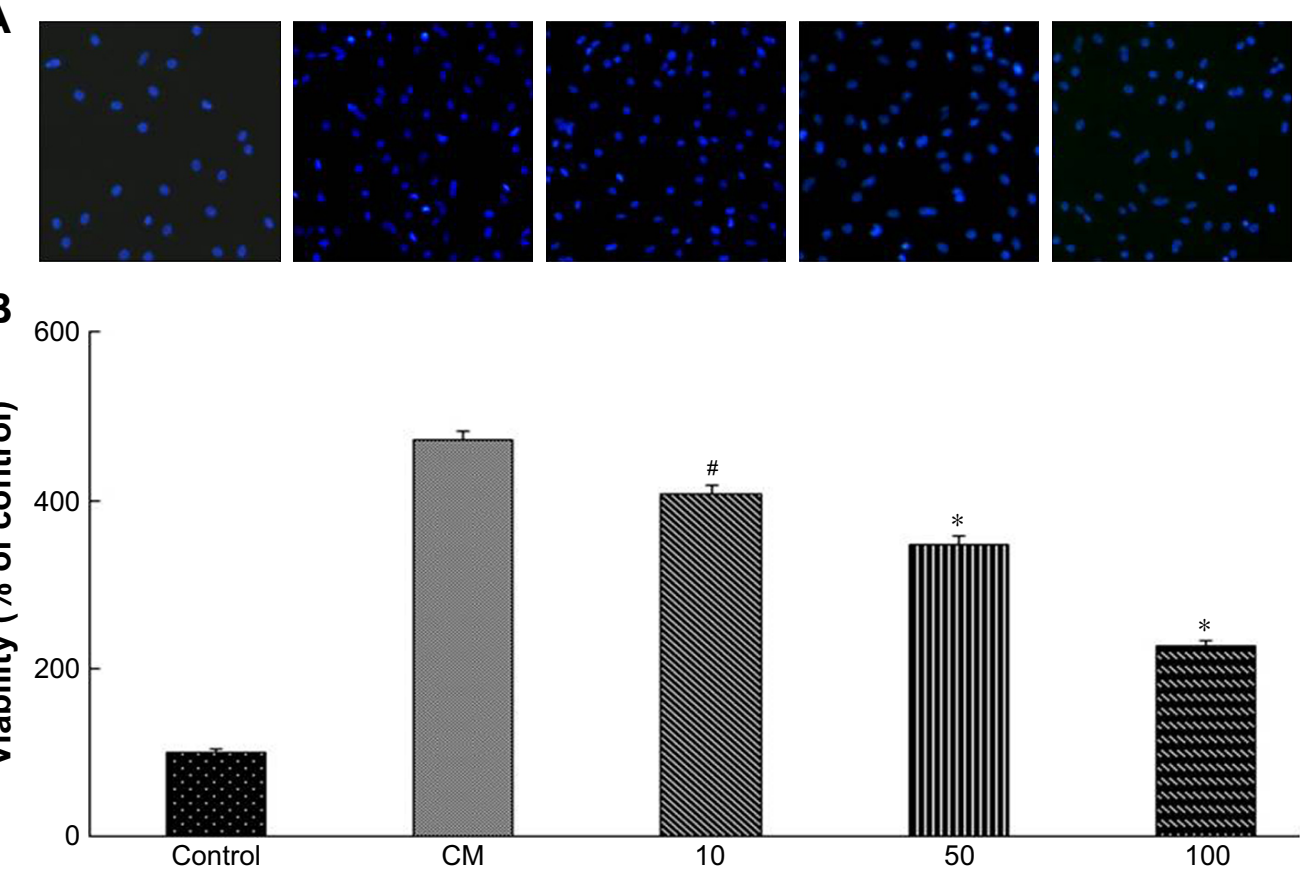

\#

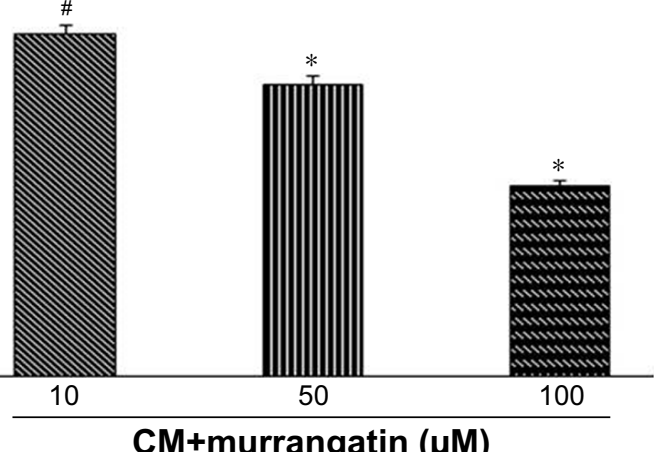

Figure 4 Murrangatin inhibited conditioned media-induced cell proliferation of HUVECs. MTT assay was performed on conditioned media-induced cell proliferation of HUVECs after treatment with the indicated concentrations of murrangatin for $24 \mathrm{~h}(\mathbf{A})$. The inhibition in cell viability is expressed as the ratio of the absorbance in cells treated with murrangatin to control cells $(\mathbf{B})$. Data are expressed as mean \pm SEM from three independent experiments $\left({ }^{*} \mathrm{CM}\right.$ vs murrangatin plus $\mathrm{CM}, p<0.05 ; * \mathrm{CM}$ vs murrangatin plus CM, $p<0.0 \mathrm{I})$.

Abbreviations: HUVECs, human umbilical vein endothelial cells; SEM, standard error of the mean; CM, conditioned medium. 
reduced in a dose-dependent manner following treatment with murrangatin, with $13.3 \%, 26.2 \%$, and $51.8 \%$ reduction relative to the control achieved with $\mathrm{CM}$ plus murrangatin at 10,50 , and $100 \mu \mathrm{M}$, respectively.

\section{Murrangatin attenuated the CM-induced angiogenic phenotype of HUVECs}

We further examined the effects of murrangatin on the physiologic events of angiogenesis, including migration, invasion, and tube formation. The wound-healing assay was used to investigate the effect of 10,50 , and $100 \mu \mathrm{M}$ murrangatin on migration in CM-treated HUVECs. As shown in Figure 5, murrangatin significantly prevented cell migration by $6.7 \%$, $16.6 \%$, and $65.4 \%$, respectively, relative to controls.

The transwell invasion assay was used to examine the effect of murrangatin on CM-induced HUVEC invasion. Murrangatin was added to the upper chamber in $0.1 \%$ endothelial basal medium, and CM was added to the lower chamber to induce cellular invasion through the membrane. Murrangatin at 10, 50, and $100 \mu \mathrm{M}$ significantly reduced CM-induced invasion of HUVECs by $8.9 \%, 19.6 \%$, and $62.9 \%$, respectively, relative to controls (Figure 6).
The tube formation assay was used to determine the effect of murrangatin on $\mathrm{CM}$-induced tube formation. $\mathrm{CM}$ induced the robust formation of tubular structures, but in contrast preincubation with murrangatin significantly and dose-dependently reduced CM-induced tube formation (Figure 7).

\section{Murrangatin inhibited angiogenesis through modulation of the AKT signaling pathway}

Activation of AKT and ERK 1/2 plays a critical role in CMinduced angiogenesis. Western blotting was used to examine the effect of murrangatin on the CM-induced phosphorylation of AKT and ERK 1/2. Murrangatin significantly attenuated CM-triggered AKT phosphorylation (Ser473), but not CMinduced ERK 1/2 phosphorylation (Figure 8). This suggests that murrangatin suppresses CM-induced angiogenesis through inhibition of the AKT signaling pathway.

\section{Discussion}

In the present study, we examined the anti-angiogenic effects of murrangatin in zebrafish embryos and human
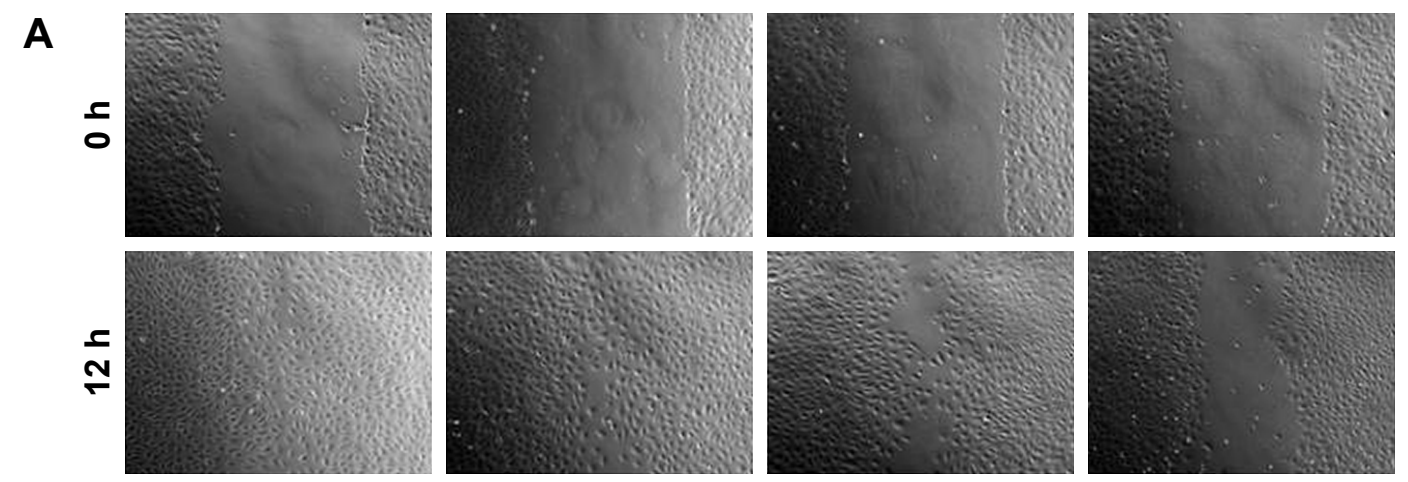

B

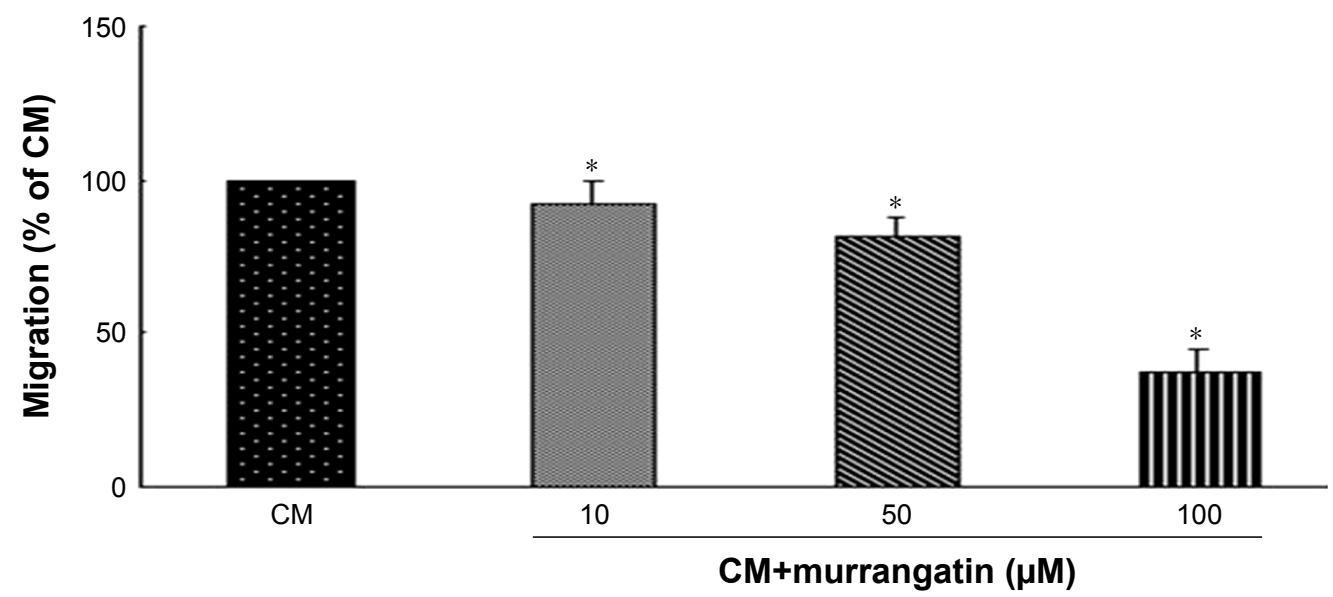

Figure 5 Anti-angiogenic effect of murrangatin in migration of HUVECs. Representative fluorescence microscopy images are shown (A). The bar chart shows quantitative data for HUVECs invasion with different treatments (B) (*CM vs murrangatin plus CM, $p<0.01$ ).

Abbreviations: HUVECs, human umbilical vein endothelial cells; CM, conditioned medium. 
A

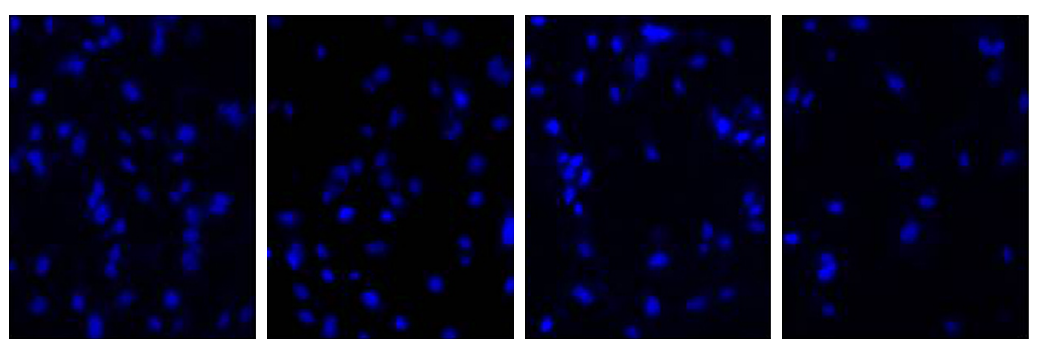

B

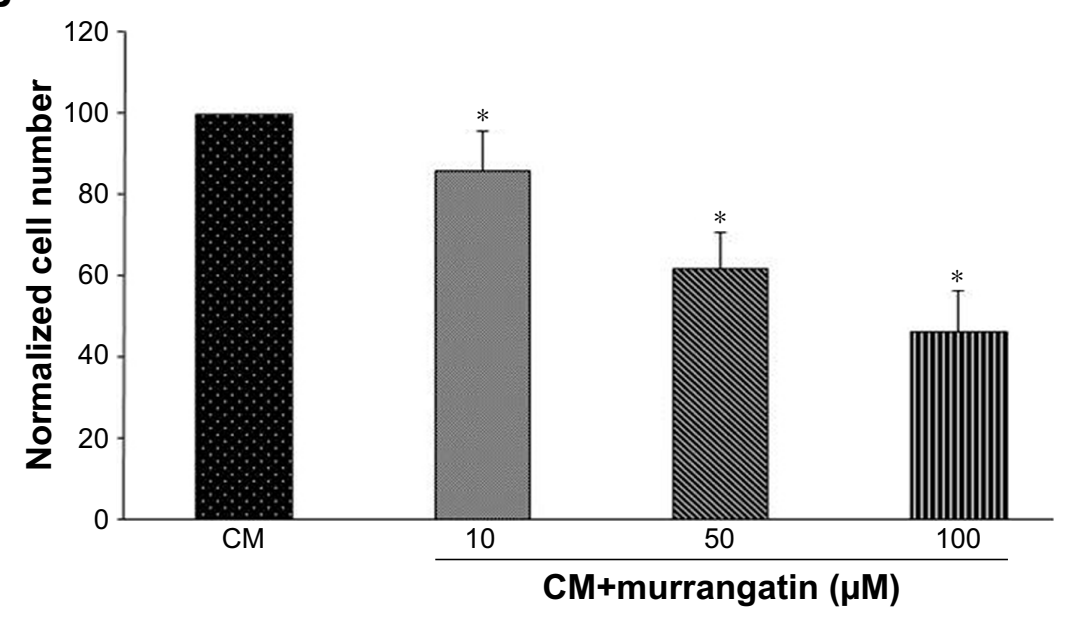

Figure 6 Anti-angiogenic effect of murrangatin in invasion of HUVECs. Representative fluorescence microscopy images are shown (A). The bar chart shows quantitative data for HUVECs invasion with different treatments (B) (*CM vs murrangatin plus CM, $p<0.01$ ).

Abbreviations: HUVECs, human umbilical vein endothelial cells; CM, conditioned medium.

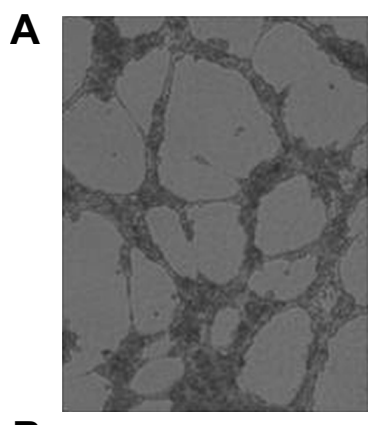

B

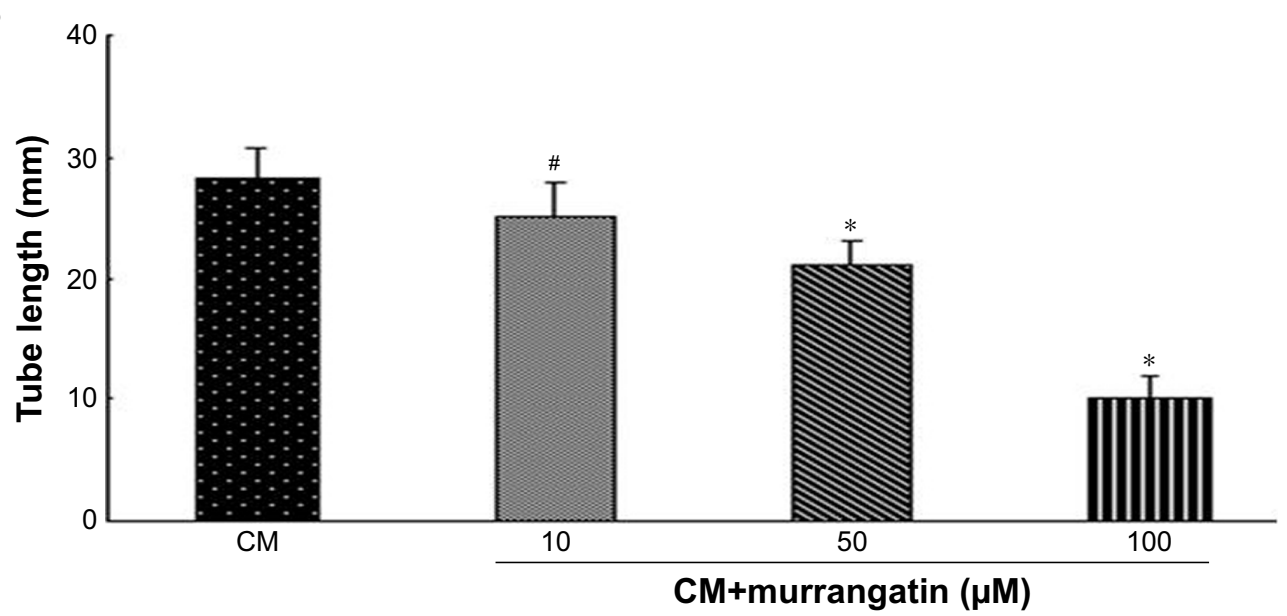

Figure 7 Anti-angiogenic effect of murrangatin in tube formation of HUVECs. Representative fluorescence microscopy images are shown (A). The bar chart shows quantitative data for HUVECs tube formation with different treatments (B) ( ${ }^{*} \mathrm{CM}$ vs murrangatin plus $\mathrm{CM}, p<0.05 ; * \mathrm{CM}$ vs murrangatin plus $\mathrm{CM}$, $p<0.0 \mathrm{I}$ ). Abbreviations: HUVECs, human umbilical vein endothelial cells; CM, conditioned medium. 
A

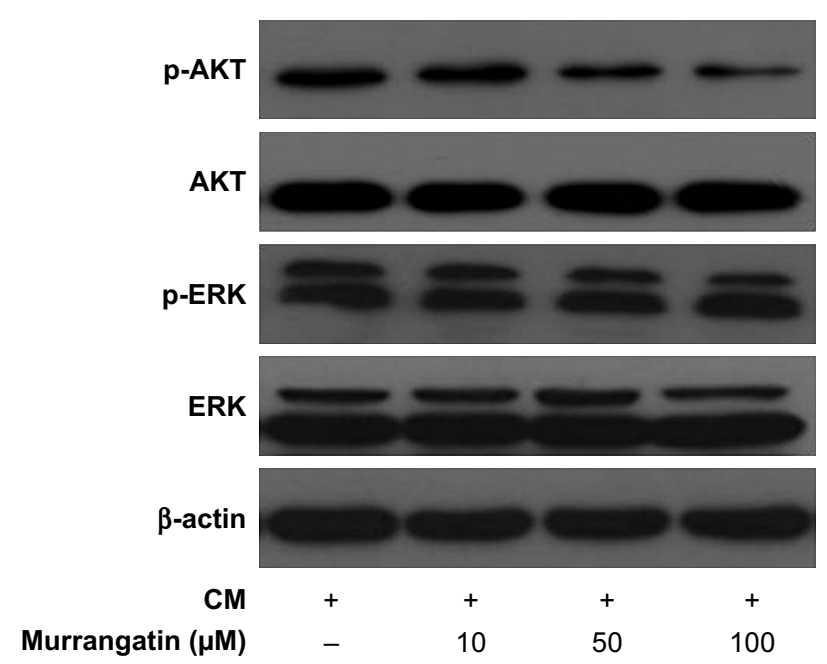

B

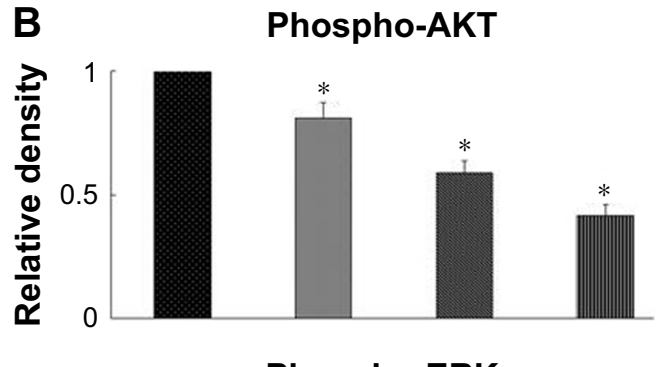

Phospho-ERK

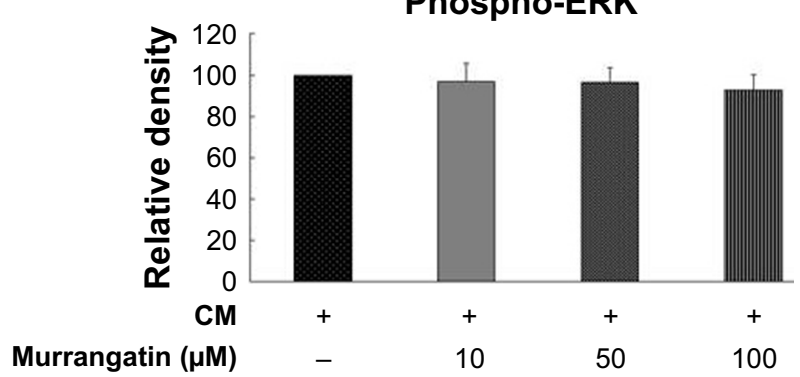

Figure 8 Murrangatin decreases phosphorylation of AKT in HUVECs. Murrangatin significantly and concentration-dependently suppressed the C-triggered phosphorylations of AKT, whereas it did not inhibit CM-induced phosphorylation of ERK I/2 in HUVECs $(\mathbf{A}$ and $\mathbf{B})\left({ }^{*} \mathrm{CM}\right.$ vs catunaregin plus CM, $\left.p<0.0 \mathrm{I}\right)$.

Abbreviations: HUVECs, human umbilical vein endothelial cells; CM, conditioned medium; ERK, extracellular signal-regulated kinase.

endothelial cells. We found that murrangatin strongly inhibited the growth of SIVs of zebrafish embryos and significantly inhibited tumor cell-induced angiogenesis in HUVECs. Murrangatin also significantly reduced tumor cell-induced activation of AKT as evidenced by the decrease in AKT phosphorylation at site Ser473. Given the importance of AKT in the pathogenesis of lung cancer, murrangatin may be a potential candidate for the development of new antilung-cancer drugs.

Blood supply is necessary for tumors to grow and metastasize. To obtain a sufficient blood supply, tumor cells secrete pro-angiogenic factors to stimulate angiogenesis. ${ }^{11,12}$ Indeed, NSCLC cells have been reported to secrete vascular endothelial growth factor to promote angiogenesis. ${ }^{13}$ However, tumor tissues also produce metabolic toxins to inhibit angiogenesis, and the balance between angiogenic and anti-angiogenic factors is thought to determine the extent of tumor angiogenesis. To investigate the potential role of murrangatin in tumor angiogensis, we first examined its effects in a transgenic zebrafish model. We found that murrangatin significantly inhibited angiogenesis during zebrafish development and that it appeared to have direct anti-angiogenic effects. The proliferation of endothelial cells is essential in tumor-induced angiogenesis, so to mimic this we used CM from A549 cell cultures to stimulate the different angiogenic phenotypes of HUVECs including cell invasion, migration, and tube formation. We demonstrated that murrangatin significantly inhibited the CM-induced angiogenic phenotypes of HUVECs in vitro.
To further investigate the underlying mechanisms by which murrangatin inhibited CM-induced angiogenesis, we examined angiogenesis-related AKT and ERK signaling pathways in HUVECs receiving murrangatin. We found that murrangatin inhibited AKT phosphorylation at site Ser473, which has been linked to the promotion of angiogenesis. ${ }^{14-16}$ Thus, we identified the inhibition of AKT-associated angiogenesis as a novel biological function of murrangatin. Given that AKT signaling pathways play a critical role in NSCLC, our results indicate that murrangatin has the potential to be developed as an anti-lung-cancer drug.

\section{Conclusion}

The present study showed that murrangatin had potent inhibitory action on the SIV growth of transgenic zebrafish embryos and could suppress CM-induced different angiogenic phenotypes of HUVECs. The anti-angiogenesis was shown to be mediated by AKT inhibition. Because AKT signaling pathways have a critical role in lung cancer, our results, together with previous observations, indicate that murrangatin is a potential candidate for the treatment of lung cancer.

\section{Acknowledgment}

This study was supported by grants from the National Natural Science Foundation of China (nos. 31670349 and 41230962).

\section{Disclosure}

The authors report no conflicts of interest in this work. 


\section{References}

1. Hoffman PC, Mauer AM, Vokes EE. Lung cancer. Lancet. 2000; 355(9202):479-485.

2. Macchiarini P, Fontanini G, Hardin MJ, et al. Relation of neovascularisation to metastasis of non-small-cell lung cancer. Lancet. 1992; 340(8812):145-146.

3. Jiang BH, Liu LZ. PI3K/PTEN signaling in angiogenesis and tumorigenesis. Adv Cancer Res. 2009;102:19-65.

4. Zhang Z, Neiva KG, Lingen MW, et al. VEGF-dependent tumor angiogenesis requires inverse and reciprocal regulation of VEGFR1 and VEGFR2. Cell Death Differ. 2010;17(3):499-512.

5. You CX, Yang K, Wang CF, et al. Cytotoxic compounds isolated from Murraya tetramera Huang. Molecules. 2014;27;19(9):13225-13234.

6. Lu XL, Luo D, Yao XL, et al. Dl-3n butylphthalide promotes angiogenesis via the extracellular signal-regulated kinase $1 / 2$ and phosphatidylinositol 3-kinase/Akt-endothelial nitric oxide synthase signaling pathways. $J$ Cardiovasc Pharmacol. 2012;59(4):352-362.

7. Lam KH, Alex D, Lam IK, et al. Nobiletin, a polymethoxylated flavonoid from citrus, shows anti-angiogenic activity in a zebrafish in vivo model and HUVEC in vitro model. $J$ Cell Biochem. 2011;112(11): 3313-3321.

8. Lu XL, Xu ZL, Yao XLi, et al. Marine cyclotripeptide X-13 promotes angiogenesis in zebrafish and human endothelial cells via PI3K/Akt/ eNOS signaling pathways. Mar Drugs. 2012;10(6):1307-1320.
9. Cai W, Wang M, Moore P, et al. The novel proangiogenic effect of hydrogen sulfide is dependent on Akt phosphorylation. Cardiovasc Res. 2007;76(1):29-40.

10. Chen KS, Wu CC, Chang FR, et al. Bioactive coumarins from the leaves of Murraya omphalocarpa. Planta Med. 2003;69(7):654-657.

11. Liao D, Johnson RS. Hypoxia: a key regulator of angiogenesis in cancer. Cancer Metastasis Rev. 2007;26(2):281-290.

12. Carmeliet P. Angiogenesis in life, disease and medicine. Nature. 2005; 438(7070):932-736.

13. Chen JJ, Yao PL, Yuan A, et al. Up-regulation of tumor interleukin-8 expression by infiltrating macrophages: its correlation with tumor angiogenesis and patient survival in non-small cell lung cancer. Clin Cancer Res. 2003;9(2):729-737.

14. Shaulian E, Karin. MAP-1 as a regulator of cell life and death. Nat Cell Biol. 2002;4(5):E131-E136.

15. Dummler B, Hemmings BA. Physiological roles of PKB/Akt isoforms in development and disease. Biochem Soc Trans. 2007;35(PT2): 231-235.

16. Zhang Y, Wang L, Zhang M, et al. Potential mechanism of interleukin-8 production from lung cancer cells: an involvement of EGF-EGFR-PI3KAkt-Erk pathway. Cell Physiol. 2012;227(1):35-43.

\section{Publish your work in this journal}

Drug Design, Development and Therapy is an international, peerreviewed open-access journal that spans the spectrum of drug design and development through to clinical applications. Clinical outcomes, patient safety, and programs for the development and effective, safe, and sustained use of medicines are the features of the journal, which has also been accepted for indexing on PubMed Central. The manuscript management system is completely online and includes a very quick and fair peer-review system, which is all easy to use. Visit http://www.dovepress.com/testimonials.php to read real quotes from published authors.

Submit your manuscript here: http://www.dovepress.com/drug-design-development-and-therapy-journal 